\title{
Understanding Reasons for Treatment Discontinuation, Attitudes and Education Needs Among People Who Discontinue Type 2 Diabetes Treatment: Results from an Online Patient Survey in the USA and UK
}

Aude Roborel de Climens · Emilie Pain · Anders Boss •

Alka Shaunik

Received: April 24, 2020 / Published online: June 12, 2020

(C) The Author(s) 2020

\section{ABSTRACT}

Introduction: Type 2 diabetes mellitus (T2DM) requires long-term treatment to achieve and maintain glycaemic control; however, up to $50 \%$ of people with T2DM discontinue treatment by 1 year. It is therefore important to understand the patient perspective of therapeutic adherence and persistence.

Methods: An online questionnaire was presented to people with T2DM in the USA and UK on PatientLive ${ }^{\circledR}$, a platform of Carenity, an online patient community. Those who discontinued at least one T2DM treatment within the last 6 months answered open-ended questions aimed to assess the reasons for discontinuation, how discontinuation could have been prevented, and what would have improved the experience with the discontinued treatment. Thematic qualitative analysis was performed on respondents' answers to these questions.

Digital features To view enhanced digital features for this article go to https://doi.org/10.6084/m9.figshare. 12301961.

A. Roborel de Climens ( $\square)$

Sanofi, Lyon, France

e-mail: aude.roborel-de-climens@sanofi.com

E. Pain

Carenity, Paris, France

A. Boss - A. Shaunik

Sanofi, Bridgewater, NJ, USA
Results: Oral antidiabetics were the most commonly discontinued treatments $(93 / 161)$, followed by insulin (40/161) and glucagon-like peptide 1 receptor agonists (13/161). Main reasons for treatment discontinuation overall were side effects (57/161), mostly gastrointestinal side effects and weight gain. The second most reported reason was drug efficacy issues (42/161). Key factors stated to prevent discontinuation were an improved care pathway (45/161) and more efficacious treatments with fewer side effects $(41 / 161)$. In the USA, treatment cost played an important role in discontinuation (14/89) and discontinuation prevention (12/89). More information about T2DM and associated treatments (56/161), help with T2DM management (24/161), and increased and informative patient-physician interaction (12/161) would have been helpful for many respondents in both countries, while some patients noted that no additional information would have been useful to improve their understanding and experience with their T2DM treatment (64/161).

Conclusions: These results emphasise the need for focused medical education and improved communication to enhance patient experience and prevent treatment discontinuation. Understanding of attributes preferred by people with T2DM can help improve therapeutic adherence and outcomes with current medications, and guide development of future therapies. 
Keywords: Discontinuation; Injectable: insulin; Oral; Survey; Treatment; Type 2 diabetes

\section{Key Summary Points}

Why carry out this study?

Long-term treatment adherence is important to ensure good clinical management of type 2 diabetes; however, up to $50 \%$ of people with this disease discontinue treatment by 1 year.

Little is known about why people discontinue treatment for type 2 diabetes and the experiences that lead to discontinuation.

This study aimed to better understand the reasons for treatment discontinuation among people with type 2 diabetes in the USA and UK, including the treatments people discontinue, their reasons for discontinuing treatment and any factors which might have prevented treatment discontinuation.

\section{What was learned from the study?}

The main reasons for treatment discontinuation overall were side effectsmostly gastrointestinal and weight gainfollowed by treatment efficacy issues, while the main factors that would have prevented discontinuation were more efficacious treatments with fewer side effects and an improved care pathway.

Our results emphasise the need for more focused medical education and improved communication to help improve therapeutic adherence, and highlight the importance of understanding patient preferences when prescribing existing medications and developing new therapies.

\section{INTRODUCTION}

Diabetes affects approximately 463 million people worldwide, of whom $90 \%$ have type 2 diabetes mellitus (T2DM) [1]. In the USA in 2018, 26.8 million adults were living with diagnosed diabetes and a further 7.3 million were estimated to have undiagnosed diabetes [2]. In the UK in 2019, there were 3.9 million people living with diagnosed diabetes [3]. As T2DM is a chronic disease, long-term treatment adherence is important to ensure good clinical management [4]. Up to $50 \%$ of people with T2DM discontinue treatment by 1 year [5].

Lack of adherence and persistence with treatment in T2DM acts as a barrier to achieving glycaemic control [6], increases the risk of microvascular and macrovascular complications [7], and increases healthcare costs [5, 8]. Patient-related factors that contribute to poor adherence are perceived treatment efficacy, fear of hypoglycaemia, the complexity and burden of treatment regimens and inconvenience associated with treatment, cost, and lack of diabetes knowledge [5]. However, little is known about why people discontinue treatment for T2DM and the experiences that lead to discontinuation [4]. An understanding of treatment and care preferences is important when currently available diabetes treatments are being prescribed or when new therapies are being developed.

This study aimed to better understand why treatment discontinuation occurs among people with T2DM in the USA and UK by conducting an online study on Carenity, a global patient community.

\section{METHODS}

\section{Study Design and Participants}

An online questionnaire was presented to people with T2DM in the USA and UK on PatientLive $^{\circledR}$, a platform of Carenity, a global online patient community. The questionnaire was designed by Sanofi and validated by Carenity. People registered on the Carenity 
T2DM community in the USA and UK were invited to participate in the survey via email.

Participants were included if they were an adult (at least 18 years of age) with T2DM and had stopped a T2DM treatment within the past 6 months. As the survey was an ad hoc (not systematic) opinion survey and no personal data were processed, ethics committee approval was not required in either the USA or the UK. For the USA, only research involving human subjects should be submitted for approval, which is not applicable for the present study. Confirmation that ethics committee approval was not required in the UK was obtained via the UK Health Research Authority website (https:// www.hra-decisiontools.org.uk/ethics/). Participant consent was obtained online at the time of registration to Carenity, prior to survey completion.

\section{Outcomes}

Participants were asked to name the T2DM treatment that they had discontinued in the last 6 months. They were then asked one closedended and three open-ended questions to determine the reasons why they originally initiated the now-discontinued treatment, the reasons for them discontinuing the treatment, how discontinuation could have been prevented, and what additional information would have improved the experience with the discontinued treatment.

\section{Data Analysis}

A sample size of 100 participants by geographic area was initially targeted. Data were collected for 98 respondents in the USA and 90 respondents in the UK. Thematic qualitative analysis was performed on respondents' answers to the open-ended questions. Cross-analysis between type of treatments and other respondents' answers was also performed. Excel ${ }^{\circledR} 2013$ was used for all data processing and analyses.

\section{RESULTS}

\section{Participant Disposition}

Of the 188 respondents to the survey, 161 responded that they had stopped a T2DM treatment in the past 6 months and were eligible to complete the whole survey. Of these 161 respondents, 89 were from the USA and 72 from the UK.

\section{Treatments Discontinued}

The majority of respondents had discontinued one treatment in the past 6 months, except six respondents who reported discontinuing two treatments. The most commonly discontinued treatments spontaneously mentioned by participants were oral antidiabetic drugs (OADs; 93/161 [58\%]), and among those who discontinued OADs, metformin was the most common OAD discontinued (55/93 [59\%]), followed by sitagliptin (12/93 [13\%]) and glipizide (7/93 [8\%]). Sodium-glucose linked transporter 2 inhibitors (SGLT2is) were discontinued in 5/161 (3\%) participants. Injectable antidiabetic therapies were also commonly discontinued (53/161 [33\%]), with insulin (40/161 [25\%]) being the most commonly discontinued followed by glucagon-like peptide-1 receptor agonists (GLP-1 RAs; $13 / 161$ [8\%]). In total, 12 (7\%) respondents who stopped taking a treatment in the past 6 months did not name the treatment, while one respondent reported discontinuing a glucose-monitoring system and one did not provide detail beyond that it was inhibitors.

OADs and insulin were the main T2DM treatments discontinued regardless of country of residence.

Data on length of time the respondent spent on treatment prior to discontinuation was spontaneously reported by 34 (21\%) respondents; 17 (50\%) of these respondents reported discontinuing treatment within the first year and $68 \%(23 / 34)$ reported having taken the treatment for less than 2 years. 


\section{Reasons for Initiating Treatment}

The main reasons given for treatment initiation are shown in Fig. 1. The most common reason reported by participants for treatment initiation was because their general practitioner or diabetologist $(69 / 161$ [43\%]) prescribed the treatment, irrespective of country and treatment initiated. The second most common reason was uncontrolled HbA1c, affecting 32\% (52/161) of all respondents, $37 \%(33 / 89)$ of US respondents and $26 \%(19 / 72)$ of UK respondents.

\section{Reasons for Discontinuing Treatment}

The main reasons given for treatment discontinuation are shown in Fig. 2. Overall, for all

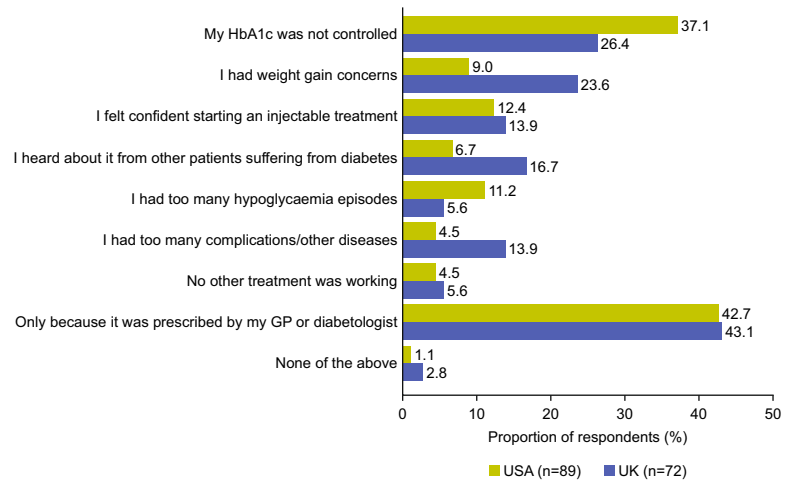

Fig. 1 Reasons for treatment initiation among respondents with type 2 diabetes in the USA and UK. GP general practitioner, HbAlc glycated haemoglobin

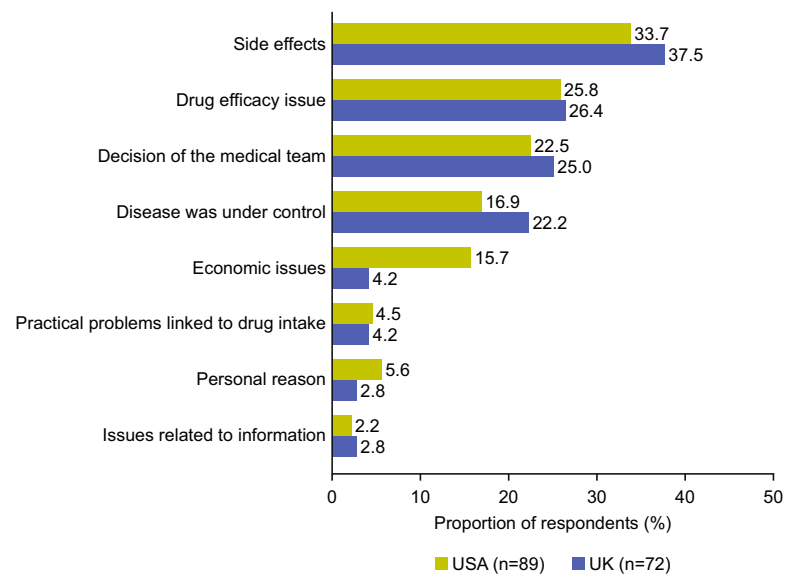

Fig. 2 Reasons for treatment discontinuation among respondents with type 2 diabetes in the USA and UK respondents and most of the treatments, side effects were the most common reason reported for treatment discontinuation (57/161 [35\%]), primarily gastrointestinal disorders $(10 / 57$ [18\%]), weight gain (10/57 [18\%]), and hypoglycaemia $(9 / 57$ [16\%]).

Drug efficacy issues (42/161 [26\%]; mainly no perceived positive effect $15 / 42$ [36\%]) and decision of the medical team (38/161 [24\%]) were also common reasons reported for discontinuation. Of those who discontinued as a result of a decision of the medical team, 20/38 (53\%) reported stopping their medication while $18 / 38(47 \%)$ reported changing their medication $(3 / 38$ [8\%] to injectable medication, $2 / 38$ [5\%] to oral medication, $1 / 38$ [3\%] to a different type of insulin, and $12 / 38$ [32\%] to an unspecified medication). Importantly, $19 \% \quad(31 / 161)$ of respondents stated that they discontinued treatment because their disease was under control.

The reasons given for treatment discontinuation differed by type of treatment. Side effects were reported as the most common reason for discontinuation of OADs (35/93 [38\%]), followed by decision of the medical team $(24 / 93$ [26\%]) and drug efficacy issues (22/93 [24\%]). Side effects were also the most common reason for discontinuation of insulin (11/40 [28\%]), followed by drug efficacy issues and decision of the medical team (each reported by 10/40 [25\%] respondents). Side effects, drug efficacy issues, and decision of the medical team were reported equally as the reason for discontinuing SGLT2is (each reported by $2 / 5$ [40\%] respondents), and drug efficacy issues were the most commonly reported reason for discontinuation of GLP-1 RAs (5/13 [38\%]), followed by side effects $(4 / 13$ [31\%]). The reason for treatment discontinuation did not tend to depend on the country of residence, except for economic issues, which were reported by $14 / 89$ (16\%) US respondents but only 3/72 (4\%) UK respondents.

\section{Factors to Prevent Treatment Discontinuation}

The key factors that would have prevented discontinuation are shown in Fig. 3. An improved care pathway $(45 / 161[28 \%])$, such as more 


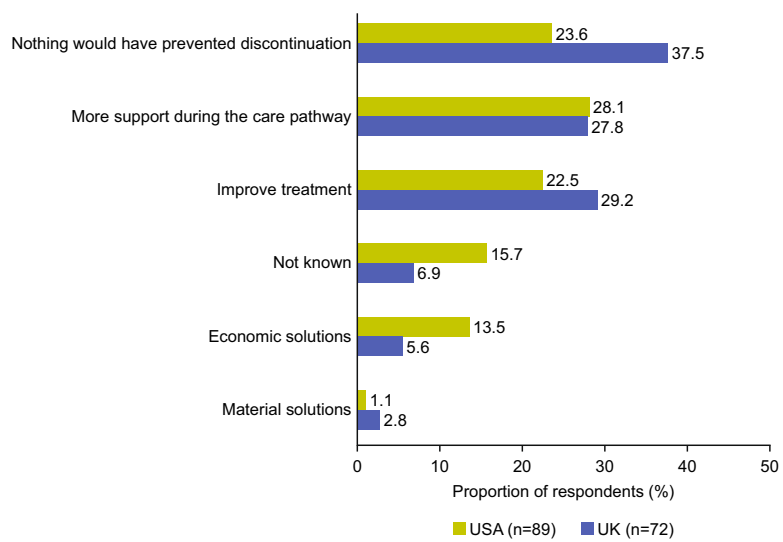

Fig. 3 Factors that would have prevented treatment discontinuation among respondents with type 2 diabetes in the USA and UK

support and information from the healthcare professional (HCP; $12 / 45$ [27\%]) and better drug dosage $(8 / 45[18 \%])$, was the main factor reported that would have prevented discontinuation. Many respondents also reported more efficacious treatments with fewer side effects (41/161 [25\%]), and economic solutions (16/161 [10\%]) as factors that would have prevented discontinuation. However, 48/161 (30\%) respondents mentioned that nothing could have prevented them from stopping their treatment.

When assessed by treatment group, respondents stated most often that nothing could have prevented them from discontinuing OADs (29/93 [31\%]), other injectable antidiabetics (7/13 [54\%]) and SGLT2is (2/5 [40\%]). However, for insulin, the most common factor that would have prevented discontinuation was more support during the care pathway (17/40 [43\%]). Exempting economic solutions, which was a more notable reason in the USA (12/89 [13\%]) than the UK $(4 / 72[6 \%])$, improvements that would have prevented treatment discontinuation did not differ by country.

The information that respondents reported would have been useful to improve their understanding of and their experience with their discontinued treatments is shown in Fig. 4. Medical information (56/161 [35\%]), help with disease management (24/161 [15\%]), increased and informative patient-physician interaction (12/161 [7\%]), and information

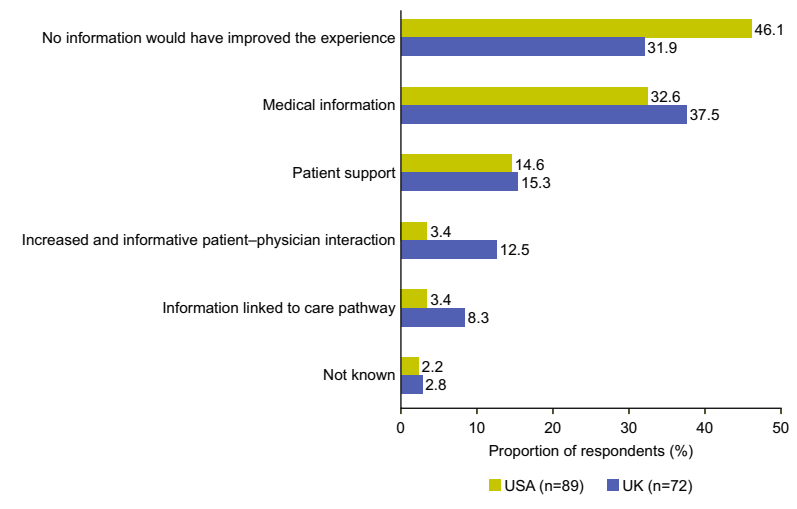

Fig. 4 Information needed to improve the treatment experience among respondents with type 2 diabetes in the USA and UK

linked to the care pathway $(9 / 161[6 \%])$ were the most needed forms of information. These responses did not differ by country of residence or treatment discontinued. Some respondents $(64 / 161[40 \%])$ said that there was no additional information that would have been useful to improve their understanding and experience of the discontinued treatment.

\section{DISCUSSION}

Good glycaemic control is essential for individuals with diabetes to avoid the risk of developing microvascular and macrovascular complications [9]. However, evidence indicates that many people with T2DM discontinue treatment within 1 year of initiation [5]. The results of this questionnaire-based study confirm these findings among people with T2DM in the USA and UK. OADs were the most commonly reported treatment discontinued, which is in line with prescription data that shows metformin to be the most commonly prescribed first-line antidiabetic treatment and sulfonylureas the most commonly prescribed secondline antidiabetic treatment [10].

Reasons for T2DM treatment discontinuation identified in the literature include perceived treatment efficacy, fear of hypoglycaemia, the complexity and burden of treatment regimens and inconvenience associated with treatment, cost, and lack of diabetes 
knowledge [5]. Other studies assessing individual preference in the treatment of T2DM typically did not fully explore the real reasons for discontinuation but focused on preferences overall through discrete-choice experiments (DCE) [11-15]. Indeed, DCE offer multiple hypothetical medication profiles for people to choose between that can differ by treatment aspects (including number of pills, oral vs injectable therapy, costs, etc.), treatment satisfaction or barriers to intensification [11-15]. The current study allowed participants to share their own response for reasons they perceived to be important specifically for improving adherence and persistence with their therapy on the basis of their experience.

Results from the present study confirm the results from previous literature [5]. Side effects, primarily gastrointestinal disorders, weight gain, and hypoglycaemia, were the main reasons overall for treatment discontinuation among the respondents of this study (35\%), indicating a need for well-tolerated treatments. About one-quarter of respondents participating in this study also reported drug efficacy issues as a cause of stopping treatment, of which no perceived positive effect was the most common reason in these respondents. However, it is important to consider that for some therapies, such as insulin, the perceived lack of efficacy may be a consequence of suboptimal titration due to patient and physician concerns such as weight gain, fear of hypoglycaemia, multiple daily injections, complexity of therapy or therapeutic burden [16, 17]. Indeed, results of an online survey in the USA, France and Germany showed that $32-42 \%$ of individuals with diabetes receiving basal insulin therapy were unaware of the need to titrate their insulin and 74\% of physicians reported fear of hypoglycaemia as a barrier to optimal titration [16]. It is important to note that another one-fifth of respondents reported that they had discontinued treatment as their disease was now under control. For various chronic conditions, a correlation has been observed between treatment adherence and perceived need (including whether an individual regarded a medication necessary, helpful, wanted, or right for them, whether they understood the drug or illness, or their condition sufficiently improved), corroborating this finding [18].

Cost was highlighted as a reason for discontinuation by $16 \%$ of US respondents, compared with only $4 \%$ of UK respondents, which could be attributed to the direct out-of-pocket costs for individuals in the USA [19] compared with costs being covered by the National Health Service in the UK [20]. In people with T2DM who discontinue or do not properly adhere to their therapy, there is an increased risk of microvascular and macrovascular complications [7], which can in turn increase healthcare costs further $[5,6]$. Therefore, affordable therapies have the potential to reduce current and future costs of living with diabetes.

In this study, the most frequently reported reason for initiating the discontinued therapy was at the guidance of the treating physician. Coupled with an improvement in the care pathway (including more information from the doctor) being reported as a key factor for preventing discontinuation in both countries, this emphasises the need for focused education and improved communication between the physician and the patient to enhance treatment understanding and experience. Current guidelines in the USA and UK stress the importance of structured and individualised education $[21,22]$ and European Association for the Study of Diabetes/American Diabetes Association guidelines state the importance of shared decision-making $[8,23]$. However, our results indicate that, despite these guidelines, individuals still perceived a need for improved care pathways and may benefit from additional information and support. Without proper communication on the rationale behind initiating therapies and shared decision-making, individuals with diabetes may not fully understand the implications of discontinuing their therapy or may be receiving therapies that they consider to be unsuitable for them, potentially making discontinuation of the treatment more likely.

While $40 \%$ of respondents reported that no information would have prevented them from discontinuing treatment, it is important to note that a third of respondents reported that medical information would have improved their experience and $15 \%$ said they would have liked 
support, stressing the importance of adequate and appropriate patient engagement. Given the number of respondents who reported initiating therapy because it was prescribed by their doctor $(43 \%)$ and not due to underlying causes, it is possible that respondents had too little information to understand why the new therapy was initiated and what should be evaluated to stop discontinuation. As such, they reported that no information would have prevented them from discontinuing treatment. Discontinuation for these participants might also have been due to burden of disease or complexity of currently available diabetes therapies. An improvement in treatment, including fewer side effects and increased efficacy, would have helped prevent treatment discontinuation in a quarter of respondents, highlighting that individualised treatment that fits the needs of each person is important among those with T2DM.

Participants in this study were recruited from a patient social platform, which has several benefits. Firstly, patients in social networks are generally more willing to share their experiences and feel free to express themselves anonymously and confidentially without their HCP's involvement, which has been shown to limit social desirability bias [24]. Secondly, such networks provide an opportunity to collect patient-reported outcomes that complement and add value to clinical data while empowering people and putting them at the centre of their own care. The use of patient platforms, however, limits the researcher's ability to include individuals who do not have access to the online tool and thus may lead to a potential recruitment bias [24]. This study captured patient-reported outcomes with no access to the participants' medical records, so it could not be confirmed whether reasons given for discontinuation were accurate (i.e. whether respondents reached glycaemic control or if the HCP had prescribed a decrease or change to their prescription). Another limitation of this study was that data were collected from a survey with a relatively small sample size. Additionally, data analyses were descriptive and variables such as education and income were not captured.

On the basis of the results of our survey, there is an opportunity to further communicate specifically on side effects and drug efficacy issues. Patient-HCP discussions before treatment initiation may help people with T2DM to better understand their options and allow for individualisation of therapy, as recommended by the American Diabetes Association/European Association for the Study of Diabetes guidelines $[8,23]$. This may lead to better patient experience, reported outcomes, and adherence. There is a wealth of patient education materials available to HCPs to help facilitate engagement with their patients.

\section{CONCLUSION}

Despite the availability of multiple classes of drugs, and multiple therapies within each drug class, glycaemic control rates remain suboptimal and a high proportion of people with T2DM do not continue on their prescribed therapies [5]. The current study provides important insights into the attitudes of people with T2DM that impact treatment adherence. The data from this survey highlight patient perspectives on reasons for treatment discontinuation among people with T2DM in the USA and UK and provide focal points for communication for these individuals when they are initiating new therapies [5]. Such communication may help more people with T2DM improve treatment adherence and persistence [5], and subsequently achieve glycaemic control and prevent short- and long-term complications. Furthermore, data from such surveys emphasise the importance of considering the perspective of individuals early in the drug development process.

\section{ACKNOWLEDGEMENTS}

The authors would like to thank the survey respondents for participating in this study.

Funding. This study was funded by Sanofi, Paris, France. The journals Rapid Service and Open Access Fees were also funded by Sanofi, Paris, France. 
Medical Writing and Editorial Assistance. Editorial assistance was provided by Tamsin Brown, MSc, and Jo Bentley, PhD, of Fishawack Communications Ltd, and was funded by Sanofi. All authors take complete responsibility for the interpretation of the data in this paper.

Authorship. All named authors meet the International Committee of Medical Journal Editors (ICMJE) criteria for authorship for this article and had full access to all the data in this study and take complete responsibility for the integrity of the data and accuracy of the data analysis. All authors participated in the interpretation of the data, the writing, reviewing, and editing of the manuscript, and had final responsibility for approving the published version.

Disclosures. Aude Roborel de Climens and Anders Boss are employees of Sanofi. Alka Shaunik was an employee of Sanofi at the time the study was conducted (current affiliation: CSL Behring, King of Prussia, PA, USA). Emilie Pain is an employee of Carenity.

Compliance with Ethics Guidelines. As the survey was an opinion survey and no personal data were processed, ethics committee approval was not required in either the USA or the UK. For the USA, only human subjects research should be submitted for approval, which is not applicable for the present study. Confirmation that ethics committee approval was not required in the UK was obtained via the UK Health Research Authority website (https:// www.hra-decisiontools.org.uk/ethics/). Participant consent was obtained online at the time of registration to Carenity, prior to survey completion.

Data Availability. The datasets generated and analysed during the current study are available from the corresponding author on reasonable request.

Open Access. This article is licensed under a Creative Commons Attribution-NonCommercial 4.0 International License, which permits any non-commercial use, sharing, adaptation, distribution and reproduction in any medium or format, as long as you give appropriate credit to the original author(s) and the source, provide a link to the Creative Commons licence, and indicate if changes were made. The images or other third party material in this article are included in the article's Creative Commons licence, unless indicated otherwise in a credit line to the material. If material is not included in the article's Creative Commons licence and your intended use is not permitted by statutory regulation or exceeds the permitted use, you will need to obtain permission directly from the copyright holder. To view a copy of this licence, visit http://creativecommons.org/licenses/by$\mathrm{nc} / 4.0 /$.

\section{REFERENCES}

1. International Diabetes Federation. IDF diabetes atlas, 9th edn. Brussels, Belgium. 2019. https:// www.diabetesatlas.org. Accessed Jan 2020.

2. CDC. National diabetes statistics report 2020. 2020. https://www.cdc.gov/diabetes/pdfs/data/statistics/ national-diabetes-statistics-report.pdf. Accessed May 2020.

3. Diabetes UK. Diabetes prevalence 2019. 2020. https://www.diabetes.org.uk/professionals/positionstatements-reports/statistics/diabetes-prevalence2019. Accessed Feb 2020.

4. Sabaté E, editor. Adherence to long-term therapies: evidence for action. Geneva: World Health Organization; 2003.

5. Polonsky WH, Henry RR. Poor medication adherence in type 2 diabetes: recognizing the scope of the problem and its key contributors. Patient Prefer Adherence. 2016;10:1299-307.

6. Guerci B, Chanan N, Kaur S, Jasso-Mosqueda JG, Lew E. Lack of treatment persistence and treatment nonadherence as barriers to glycaemic control in patients with type 2 diabetes. Diabetes Ther. 2019;10(2):437-49.

7. Fowler MJ. Microvascular and macrovascular complications of diabetes. Clin Diabetes. 2008;26(2): 77-82.

8. Davies M, D'Alessio D, Fradkin J, et al. Management of hyperglycaemic in type 2 diabetes, 2018. 
A consensus report by the American Diabetes Association (ADA) and the European Association for the Study of Diabetes (EASD). Diabetologia. 2018;61:2461-98.

9. Nathan DM, DCCT/EDIC Research Group. The diabetes control and complications trial/epidemiology of diabetes interventions and complications study at 30 years: overview. Diabetes Care. 2014;37(1):9-16.

10. Montvida O, Shaw J, Atherton J, Stringer F, Paul S. Long-term trends in antidiabetes drug usage in the U.S.: real-world evidence in patients newly diagnosed with type 2 diabetes. Diabetes Care. 2018;41: 69-78.

11. Janssen E, Longo D, Bardsley J, Bridges J. Education and patient preferences for treating type 2 diabetes: a stratified discrete-choice experiment. Patient Prefer Adherence. 2017;11:1729-36.

12. WHO. Guidelines on second- and third-line medicines and type of insulin for the control of blood glucose levels in non-pregnant adults with diabetes mellitus. World Health Organization, Geneva. 2018. Licence: CC BY-NC-SA 3.0 IGO.

13. Mansfield C, Sikirica M, Pugh A, et al. Patient preferences for attributes of type 2 diabetes mellitus medications in Germany and Spain: an online discrete-choice experiment survey. Diabetes Ther. 2017;8:1365-78.

14. Hertroijs DFL, Elissen AMJ, Brouwers MCGJ, Hiligsmann M, Schaper NC, Ruwaard D. Preferences of people with type 2 diabetes for diabetes care: a discrete choice experiment. Diabetic Med. 2019. https://doi.org/10.1111/dme.13969.

15. Fifer S, Rose J, Hamrosi KK, Swain D. Valuing injection frequency and other attributes of type 2 diabetes treatments in Australia: a discrete choice experiment. BMC Health Serv Res. 2018;18(1):675.

16. Berard L, Bonnemaire M, Mical M, Edelman S. Insights into optimal basal insulin titration in type 2 diabetes: results of a quantitative survey. Diabetes Obes Metab. 2018;20(2):301-8.

17. Russell-Jones D, Pouwer F, Khunti K. Identification of barriers to insulin therapy and approaches to overcoming them. Diabetes Obes Metab. 2018;20(3):488-96.

18. Gadkari AS, McHorney CA. Medication nonfulfillment rates and reasons: narrative systematic review. Curr Med Res Opin. 2010;26(3):683-705.

19. American Diabetes Association. Economic costs of diabetes in the U.S. in 2017. Diabetes Care. 2018;41(5):917-28.

20. Diabetes.co.uk. Cost of Diabetes. 2019. https:// www.diabetes.co.uk/cost-of-diabetes.html. Accessed Feb 2020.

21. Wong W. Exploring the potential of clinical pathways for diabetes. J Clinical Pathways. 2017;2(Suppl 1):S5-6.

22. NHS RightCare Pathway: diabetes. 2018. https:// www.england.nhs.uk/rightcare/wp-content/uploads/ sites/40/2018/07/nhs-rightcare-pathway-diabetes.pdf. Accessed Mar 2020.

23. American Diabetes Association. Standards of Medical Care in Diabetes - 2020. Diabetes Care. 2020;43(Supplement 1):S1-212.

24. Ravoire S, Lang M, Perrin E, et al. Advantages and limitations of online communities of patients for research on health products. Therapie. 2017;72: $135-43$ 